\title{
VITA - Estimando a satisfação de estudantes por meio da Análise de Sentimentos
}

\author{
Leonardo Preuss $^{1}$, Joel dos Santos ${ }^{1}$, Gustavo Paiva Guedes ${ }^{1}$ \\ ${ }^{1}$ Escola de Informática e Computação - CEFET/RJ
}

\begin{abstract}
Resumo. Universidades públicas ao redor do mundo enfrentam um grave e recorrente problema: o alto índice de evasão de alunos em cursos de graduação. Excluindo-se questões vocacionais e financeiras, diversas causas são apontadas para tal problema, como a qualidade docente, o baixo desempenho em disciplinas básicas, e também a insatisfação discente, sendo um indicador altamente responsável por sua evasão em curso de nível superior. Nesse sentido, este trabalho propõe um sistema para melhoria na interação aluno-professor, de modo que o docente tenha maior facilidade em identificar e solucionar os problemas que respondem pela insatisfação do aluno. Resultados preliminares indicam sucesso na melhoria da percepção do professor para com o aluno.
\end{abstract}

\begin{abstract}
Public universities around the world face a serious and recurring problem: the high dropout rate of undergraduate students. Besides vocational and financial issues, several causes were pointed to such problem, like the teaching quality, low performance on basic disciplines and also the student dissatisfaction, which is a highly responsible indicator for its evasion on higher education. In this sense, the current work proposes a platform for improving studentteacher interaction easying the teacher identification and solving of problems that responds to student's dissatisfaction. Preliminary results demonstrate success on improving teacher's perception about the student.
\end{abstract}

\section{Introdução}

Pesquisas na área da educação [Chen et al. 2009, Weerasinghe et al. 2017, Elliott and Shin 2002], indicam que o nível de satisfação do estudante é um importante indicador na medida do sucesso de uma universidade. Estudantes com altos níveis de satisfação são mais propensos a continuar no curso [Seldin 1993], em contraste àqueles que estão insatisfeitos. Além disso, a qualidade docente e do método de ensino são considerados fatores cruciais e altamente responsáveis na satisfação dos alunos [de Souza and Reinert 2010].

Assim, diversos problemas relacionados ao ensino público no Brasil e no mundo, são motivados pela insatisfação dos alunos diante do curso, que geram altos impactos no índice de evasões nas instituições de ensino. Esse é um grave problema e traz grandes desperdícios de recursos às universidades [Vallejos and Steel 2014], já que uma grande quantidade de verbas necessárias para sustentar um aluno na universidade são aplicadas sem retorno na preparação desse. Acresce-se a isso que os índices de evasão estão intimamente relacionados com o baixo desempenho acadêmico em disciplinas iniciais, muitas vezes causados pela falta de informação sobre as dificuldades e necessidades dos alunos perante a disciplina [Manhães et al. 2011]. Tal cenário se agrava em turmas com 
VII Congresso Brasileiro de Informática na Educação (CBIE 2018)

Anais do XXIX Simpósio Brasileiro de Informática na Educação (SBIE 2018)

um grande número alunos, tornando-se mais complicado para o professor acompanhá-los individualmente.

Por este motivo, torna-se de grande valia a criação de um mecanismo capaz de melhorar a percepção do professor a respeito dos alunos. Isso permite que o professor antecipe a insatisfação dos alunos em uma disciplina e tenha maior agilidade na compreensão de suas necessidades e na adequação das aulas. Este artigo tem como objetivo propor um sistema que auxilie o professor na percepção do andamento dos alunos em suas aulas, revelando seu grau de satisfação ou insatisfação durante o período letivo. A avaliação é feita tendo como base comentários escritos sobre cada aula, fornecidos pelos alunos.

Uma vantagem da abordagem proposta é que o professor não toma ciência dos comentários fornecidos pelos alunos, apenas recebe informações da situação desses em forma de gráficos. Dessa forma, os alunos possuem maior liberdade para discorrerem sobre sua percepção das aulas. Outra vantagem oferecida está na utilização de uma abordagem computacional para avaliação dos comentários, que não se encontra sujeita a diferenças na interpretação, tal como nas avaliações tradicionais de fim de curso, em que são escritos comentários manualmente, que poderiam ser compreendidos de forma diferenciada pelo professor. Ainda, a abordagem proposta prevê a avaliação continuada, por parte dos alunos, a cada aula. Assim, permite-se que o professor observe tendências nos graus de satisfação e sentimentos predominantes dos alunos, bem como a recomendação de ajustes pela Comissão Própria de Avaliação ao longo do semestre. É também importante ressaltar que essa abordagem permite uma análise mais fiel em relação a abordagem tradicional, em que os comentários se destinam a uma visão geral do curso. Além disso, a ferramenta proposta torna-se bastante adequada em turmas de grande porte, já que a percepção da satisfação, do ponto de vista do professor, é degradada pela quantidade de alunos. Outra aplicação possível é em turmas de ensino a distância nas quais o baixo contato do aluno com o professor impacta nessa percepção.

As informações utilizadas para produção dos gráficos, tem como base um módulo fundamentado na Análise de Sentimentos [Liu 2012]. Esse módulo avalia os comentários fornecidos pelos alunos a cada aula, extraindo o grau de satisfação de cada um, formando uma análise global e individual, numa abordagem similar a de trabalhos de User eXperience (UX) [Hassenzahl 2008]. A avaliação é feita com base em dicionários em que verbetes de um idioma são categorizados com base em aspectos afetivos, sociais, psicológicos e linguísticos. Na proposta apresentada neste artigo são utilizados os dicionários de léxicos afetivos LIWC (Linguistic Inquiry and Word Count) [Tausczik and Pennebaker 2010], na versão português do Brasil, e ANEW-br (Affective Norms for English Words) [Bradley et al. 1999] para o cruzamento dos dados e maior precisão na estimativa dos sentimentos e grau de satisfação encontrados. Por fim, os tipos de gráficos disponibilizados no sistema foram escolhidos mediante pesquisa com os professores a fim de se conhecer as melhores formas de visualização.

O restante deste artigo está dividido como segue. A Seção 2 fornece o referencial teórico para compreensão do trabalho proposto. A Seção 3 apresenta os trabalhos relacionados, revelando o estado da arte de trabalhos mais relevantes para o mesmo segmento, utilizados ao redor do mundo. A Seção 4 discute o modelo de avaliação proposto neste trabalho, bem como a arquitetura da implementação apresentada. A Seção 5 expõe e dis- 
VII Congresso Brasileiro de Informática na Educação (CBIE 2018)

Anais do XXIX Simpósio Brasileiro de Informática na Educação (SBIE 2018)

cute os resultados obtidos do trabalho proposto. Por fim, a Seção 6 faz as considerações finais apontando possíveis trabalhos futuros.

\section{Léxicos Afetivos}

A identificação e classificação dos sentimentos na Análise de Sentimentos é comumente realizada de duas formas: (i) por meio do aprendizado de máquina ou (ii) com o uso de dicionário de léxicos afetivos [Gonçalves et al. 2013]. No caso do aprendizado de máquina, os algoritmos mais utilizados são Naive Bayes, SVM, dentre outros. Já os dicionários de léxicos afetivos contém palavras associadas a emoções, sentimentos, processos psicológicos, dentre outros [Filho et al. 2013]. O presente trabalho faz uso dos dicionários de léxicos afetivos LIWC e ANEW, detalhados nos próximos parágrafos.

O LIWC (Linguistic Inquiry And Word Count) [Tausczik and Pennebaker 2010] é um dicionário de léxicos afetivos em que os verbetes estão associados à categorias psicológicas, afetivas, gramaticais, dentre outras. O dicionário original, em inglês, foi desenvolvido pelo psicólogo James Pennebaker [Tausczik and Pennebaker 2010], que afirma que o estilo de escrita contém informações implícitas, e a forma de se estruturar e organizar uma frase é mais informativo que o conteúdo em si. Assim, como o próprio nome diz, o LIWC é uma ferramenta para contagem de palavras e categorias. Dado um texto, realiza-se a contagem de todas as categorias identificadas, formando-se um vetor com a categoria e a frequência.

A única versão do LIWC em português do Brasil (de 2007) [Filho et al. 2013] conta com mais de 127 mil verbetes e 64 categorias. É interessante destacar que um verbete pode estar associado a mais de uma categoria. Por exemplo, a palavra "bom" está associada às categorias de emoção positiva, afeto, sentimento, etc. Por outro lado, a palavra "mau" está associada às categorias afeto, emoção negativa, raiva, dentre outras.

O ANEW (Affective Norms for English Words) [Bradley et al. 1999] é outro dicionário de léxicos afetivos. No entanto, seus verbetes estão associados aos conceitos de valência (polaridade), excitação (ativação) e dominância. Estas componentes provêm de conceitos diretamente relacionados à psicologia e dizem respeito, respectivamente, a polaridade de uma emoção (positiva ou negativa), ao grau de ativação desta emoção, e o quanto a emoção é de submissão ou dominância. No ANEW, as 3 dimensões estão compreendidas em uma escala de valores decimais com seu mínimo em 1 e máximo em 9 , contendo também seus respectivos desvios-padrão para cada palavra.

A partir da valência e excitação, é possível gerar um gráfico indicando a emoção sentida por um indivíduo. Uma forma comum de tal representação exibe a valência no eixo horizontal e a excitação no eixo vertical. Com estas informações podemos estimar, mediante o ângulo do gráfico gerado, a emoção sentida pelo usuário. Por exemplo, uma baixa excitação e uma polaridade bastante positiva, indicam um estado de calma. Já uma grande excitação e valência negativa, indicam um estado de estresse ou raiva. A Figura 1 apresenta exemplos de sentimentos que podem ser obtidos a partir dessa representação.

\section{Trabalhos Relacionados}

No contexto da Análise de Sentimentos, existem alguns trabalhos publicados na literatura voltados ao auxílio na área da educação. Dentre eles, podem-se destacar [Aung and Myo 2017, Barrón-Estrada et al. 2017, Pong-Inwong and Kaewmak 2016, 
VII Congresso Brasileiro de Informática na Educação (CBIE 2018)

Anais do XXIX Simpósio Brasileiro de Informática na Educação (SBIE 2018)

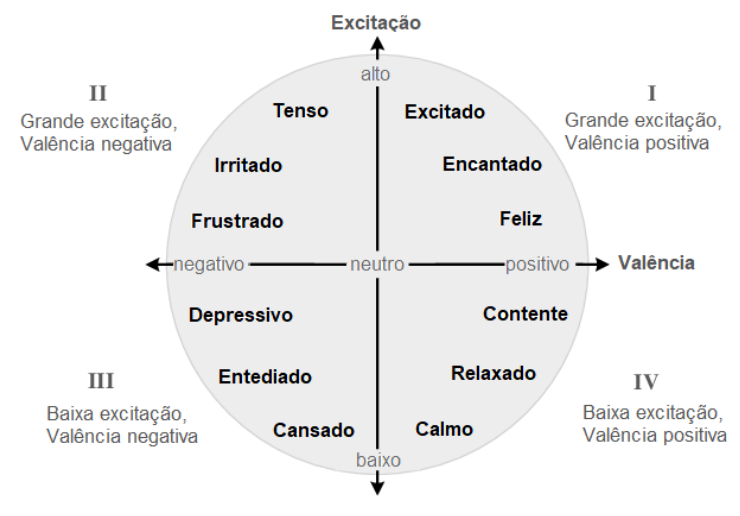

Figura 1. Modelo valência-excitação de James Russel. Cada ponto do gráfico apresenta um sentimento estimado a partir dos valores de valência e excitação. Adaptado de [Yu et al. 2016]

Balahadia et al. 2016, Rani and Kumar 2017, Ortigosa et al. 2014, Robinson et al. 2013, Rude et al. 2004].

Em [Aung and Myo 2017], os autores apresentam um sistema de análise de sentimentos em comentários de alunos sobre os professores. Esse trabalho utilizou uma abordagem léxica para a análise de sentimentos, de forma semelhante ao presente trabalho. No entanto, o dicionário utilizado para classificação das palavras é diferente do dicionário utilizado nesse trabalho. No trabalho mencionado, o resultado da avaliação do professor é expressado em 7 níveis, sendo eles: fortemente positivo, moderadamente positivo, fracamente positivo, neutro, fracamente negativo, moderadamente negativo e fortemente negativo. Um grande diferencial em relação o trabalho aqui proposto é que [Aung and Myo 2017] não classifica os comentários de acordo com as emoções presentes (ex.: felicidade, raiva, tristeza).

Em [Barrón-Estrada et al. 2017], os autores apresentam um módulo para análise de sentimentos de comentários de alunos para a API (Application Programming Interface) Sensei. Sensei é um ambiente Web que funciona como uma espécie de sistema de recomendação, fornecendo instruções para os estudantes a partir dos comentários feitos. A ideia do módulo é obter as informações dos alunos estritamente sobre os exercícios de aulas. Dessa forma, o aluno resolve os exercícios e fornece ao módulo seu comentário sobre os exercícios realizados para que os professores adaptem os exercícios conforme a necessidade e, assim, o ciclo se repete. Em [Barrón-Estrada et al. 2017], o resultado é expressado em formato de polaridade (positivo, negativo ou neutro) e também não utiliza o LIWC para classificação, apesar de utilizar uma abordagem léxica.

Em [Pong-Inwong and Kaewmak 2016], os autores desenvolvem um trabalho de análise de sentimentos para comentários de alunos sobre os professores em mídias sociais. Cada aluno faz a postagem de sua opinião sobre o professor uma vez por semana. Além disso, também é proposta a melhoria de métodos de análise de sentimentos por meio da comparação entre métodos tradicionais de aprendizado de máquina, com os chamados Ensemble Learning, no qual múltiplos métodos de aprendizado de máquina são utilizados. Os resultados indicaram que os métodos de Ensemble Learning são significativamente mais eficientes que os métodos de aprendizado de máquina tradicionais. 
VII Congresso Brasileiro de Informática na Educação (CBIE 2018)

Anais do XXIX Simpósio Brasileiro de Informática na Educação (SBIE 2018)

Em [Balahadia et al. 2016], os autores apresentam uma ferramenta para avaliação de professores por estudantes, colocando a avaliação como parte da escala de desempenho de professores. Ademais, faz também a medição do quanto uma avaliação qualitativa pode complementar uma avaliação quantitativa. Neste trabalho os resultados da análise de sentimentos só assumem dois estados: positivo ou negativo. O trabalho utiliza uma abordagem léxica para a análise de sentimentos e fornece módulos para análises estatísticas e compilação de resultado, dentre outros. Além disso, o trabalho mostra que a necessidade da tarefa de análise de sentimentos é grande no que diz respeito ao trabalho tedioso do professor em ler os comentários dos alunos. Este trabalho se diferencia ao atual na análise da polaridade, já que o trabalho aqui proposto leva em conta algumas emoções negativas.

Em [Rani and Kumar 2017], os autores desenvolvem um trabalho de Análise de Sentimentos em páginas de pesquisa de avaliação de professores para comentários de alunos. O sistema desenvolvido fornece a polaridade dos sentimentos (positivo, negativo ou neutro) além de 8 categorias de emoções (tristeza, ódio, medo, desgosto, surpresa, prazer, expectativa e confiança). A partir daí o sistema ainda calcula o grau de satisfação e insatisfação do estudante. O trabalho utiliza uma abordagem léxica, com base no dicionário NRC Emotion Lexicon, para calcular a polaridade da frase, bem como classificá-la dentre as 8 categorias de emoções. É importante ressaltar, entretanto, que o léxico utilizado neste trabalho não é voltado para o idioma português do Brasil.

\section{VITA}

Conforme apresentado anteriormente, a proposta deste artigo visa permitir a avaliação, em formato de escrita livre, das aulas lecionadas. Dessa maneira, o aluno pode discorrer sem restrições sobre cada aula ministrada. Esta proposta foi realizada na forma da ferramenta VITA. VITA permite que o professor tenha acesso ao sistema, verificando o nível de satisfação geral da turma, bem como a satisfação individual dos alunos. No estágio atual, o sistema está desenvolvido para plataforma Web e aplicativo móvel, restrito aos dispositivos Android. VITA também conta com um servidor encarregado de armazenar e produzir o resultado das avaliações fornecidas pelos alunos e um banco de dados.

\subsection{Arquitetura}

VITA segue uma arquitetura cliente-servidor. A versão aluno do cliente envia, via protocolo HTTP, o texto a ser analisado ao servidor. O servidor, por sua vez, recebe a solicitação, processa-a e armazena o resultado em um banco de dados. Em um segundo momento, caso o professor tenha a intenção de visualizar o desempenho da turma, ou de um aluno específico, a versão professor do VITA solicitará ao servidor a avaliação de sua disciplina, para determinada aula. O servidor obtém as informações da aula escolhida no banco de dados, remonta-as e as retorna ao cliente em formato JSON.

Como geralmente é feito na Mineração de Texto, o processamento realizado com o comentário do aluno para análise de sentimentos, compreende várias etapas que envolvem a extração, limpeza, correção, aplicação do modelo proposto sobre os dados, e produção do resultado. As etapas das quais VITA se utiliza para realização da análise são de tokenização, correção ortográfica, transliteração, núcleo da análise de sentimentos, produção e armazenamento do resultado na base de dados. Uma ilustração destas etapas pode ser observada na Figura 2 . 
VII Congresso Brasileiro de Informática na Educação (CBIE 2018)

Anais do XXIX Simpósio Brasileiro de Informática na Educação (SBIE 2018)

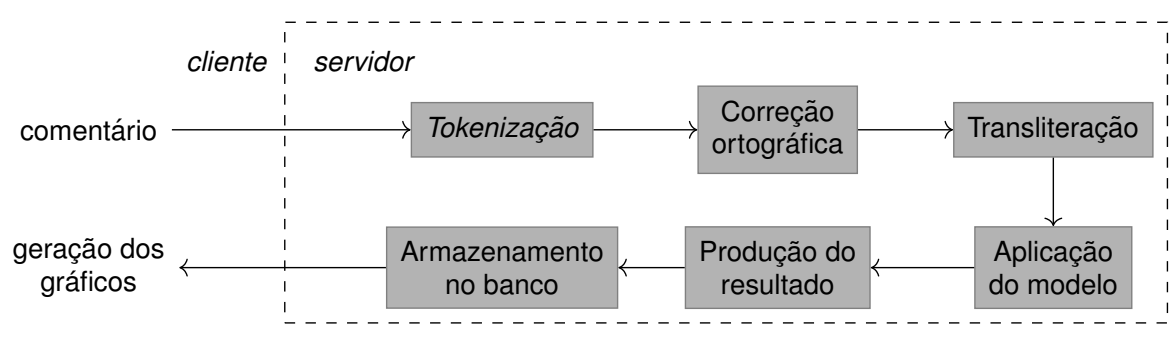

Figura 2. Etapas envolvidas no processamento dos comentários.

A etapa de tokenização identifica as palavras de um documento, por meio de espaços em branco, quebras de linha, marcas de tabulação, etc. É neste momento em que é produzido o $B O W$ (Bag-of-Words), ou seja, o conjunto completo e único de tokens, com suas respectivas frequências de repetição. A seguir, é feita a correção ortográfica das palavras, removendo possíveis erros de digitação que tenham ocorrido na escrita do texto. $\mathrm{Na}$ terceira etapa ocorre a transformação de formas abreviadas comuns de escrita (e.g., vc, tbm, blz) para a forma padrão da Língua Portuguesa. Após essas etapas, as palavras do $B O W$ estão no formato necessário para sua busca no léxico utilizado.

O resultado da aplicação do modelo é um vetor com a contagem de palavras para cada categoria do léxico utilizado. No caso deste artigo, para o cálculo da satisfação do aluno, é realizada uma filtragem das categorias de emoção positiva, emoção negativa e as subcategorias desta última, são elas: ansiedade, raiva e tristeza. O vetor produzido é finalmente armazenado no banco de dados, de modo que todas as informações sejam recuperadas posteriormente.

Quando o docente solicita a avaliação da aula, são resgatadas do banco as informações necessárias para remontar o vetor, retorná-los ao cliente, e a partir deste, produzir os gráficos a serem visualizados pelo professor. Os gráficos disponíveis são do tipo barras, setores e radar. Essa escolha foi baseada em uma pesquisa com 11 professores do curso de Ciência da Computação, utilizando o método de Condorcet [Young 1988] para seleção dos três melhores gráficos.

\subsection{Modelo}

Conforme mencionado anteriormente, o modelo proposto aqui visa estimar o nível de satisfação do aluno diante das aulas de uma disciplina. Para isso, é tomada como base a polaridade do texto escrito. Assim, este artigo propõe um modelo de avaliação dos comentários dos alunos com base nos léxicos afetivos.

O modelo aqui proposto se utiliza de uma versão adaptada do LIWC $_{\mathrm{AN}}$, uma abordagem apresentada em [Rodrigues and Guedes 2017], na qual é feita a combinação dos dicionários LIWC com o ANEW, tendo como objetivo melhorar a precisão da análise. $A$ aplicação do LIWC $_{A N}$ produz um vetor que relaciona as categorias encontradas em um texto e suas respectivas contagens. Sua diferença para o LIWC é que a união entre os dicionários acrescenta um peso aos verbetes do LIWC, de acordo com a excitação e valência de uma palavra encontrada no ANEW. Assim garante-se maior correspondência entre a polaridade das palavras.

A adaptação aqui proposta altera a forma como o pesos advindos do ANEW são calculados em [Rodrigues and Guedes 2017]. A fórmula a seguir determina o peso $p(w)$ 
VII Congresso Brasileiro de Informática na Educação (CBIE 2018)

Anais do XXIX Simpósio Brasileiro de Informática na Educação (SBIE 2018)

a ser atribuído para uma palavra $w$, com base nos valores de valência $(V(w))$ e excitação $(A(w))$ obtidos do ANEW.

$$
p(w)=\frac{|V(w)-5| \times A(w)}{36}
$$

Na fórmula apresentada na Equação 1 o valor de valência é subtraído de 5 , deslocando sua escala original $[1,9]$ para o intervalo $[-4,4]$. Assim, a neutralidade da valência fica representada pelo valor central 0 . O valor resultante é multiplicado pelo valor de excitação (A, do inglês arousal). Como a escala de excitação não possui um valor central, permanece no mesmo intervalo [1,9]. Ao final, é feita a divisão por 36 , de forma que o resultado seja um valor no intervalo $[0,1]$.

A partir do modelo bag of words (BOW) [Kao and Poteet 2007] obtido nas fases anteriores à utilização do modelo proposto nesse artigo, a aplicação do LIWC $_{\mathrm{AN}}$ é feita de acordo com a descrição que se segue. $V$ é o vetor que se espera ter como resultado, encarregado de guardar as categorias e suas respectivas frequências. Este é inicializado com um conjunto vazio, aguardando ser preenchido nos passos seguintes. Para cada palavra existente no $B O W$, é feita a busca ao LIWC por meio da função findWord, que retorna as categorias associadas àquela palavra para o vetor $C$. Após, é feito o cálculo do peso obtido no ANEW usando a Equação 1. Caso a palavra solicitada não exista no ANEW, o peso retornado é 0 , sendo apenas o peso do LIWC $\left(\mathrm{w}_{1}\right)$ acrescentado. Cabe ressaltar que esta divisão foi realizada de forma que o ANEW contribua mais que o LIWC. Assim, a parcela de peso para o LIWC foi fixada em 0.3 e o ANEW podendo alcançar até 0.7.

Após a obtenção dos valores de peso, caso alguma categoria contida em $C$ não exista no vetor $V$, esta é adicionada ao vetor $V$. Após, esta o valor armazenado nesta categoria é somada com o peso encontrado. O procedimento se repete até que todas as palavras do $B O W$ tenham sido visitadas. Ao final do procedimento, temos o vetor $V$ preenchido. Uma imagem deste vetor pode ser vista na Figura 3 .

\begin{tabular}{|c|c|c|c|c|}
\hline posemo & negemo & \multicolumn{1}{c}{ anx } & \multicolumn{1}{c}{ anger } & sad \\
\hline 35,6 & 40,1 & 10,5 & 22,7 & 18,5 \\
\hline
\end{tabular}

Figura 3. Exemplo de vetor ponderado com suas respectivas categorias

A satisfação é então calculada a partir da razão entre o valor da categoria de emoção positiva com a soma dos valores encontrados para categorias de emoção negativa. As categorias levadas em conta neste trabalho foram de emoção positiva (posemo), emoção negativa (negemo), ansiedade (anx), raiva (anger) e tristeza (sad).

É importante ressaltar que, apesar da categoria negemo contemplar as subcategorias anx, sad e anger, nem toda palavra negativa pertence a uma das três últimas categorias citadas. Portanto, a categoria de emoção negativa é contada levando em conta apenas as emoções negativas que não se encontram em suas três subcategorias. Para o exemplo da Figura 3 , a satisfação encontrada é de $35,6 /(35,6+40,1+10,5+22,7+18,5)$ resultando em 0,28 ou $28 \%$. 
VII Congresso Brasileiro de Informática na Educação (CBIE 2018)

Anais do XXIX Simpósio Brasileiro de Informática na Educação (SBIE 2018)

\subsection{Implementação}

Conforme já apresentado anteriormente, a ferramenta VITA está implementada para dispositivos Android, contando também com uma versão Web. Ambos os perfis de alunos e professores podem acessar o sistema, considerando que as opções disponíveis são distintas de acordo com o perfil de acesso. Enquanto o aluno apenas envia a avaliação, o professor apenas as visualiza. A Figura 4 mostra o layout de envio por parte do aluno. Já a Figura 5 apresenta a visão da turma pelo professor para dispositivos Android.

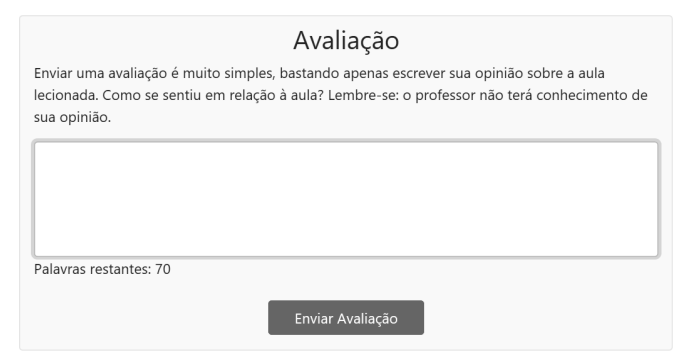

Figura 4. Versão Web para perfil aluno

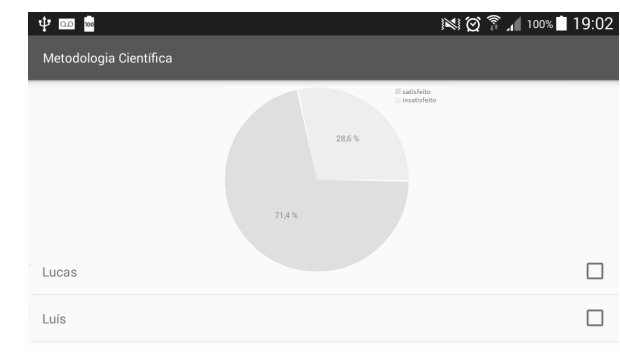

Figura 5. Versão para perfil professor.

\section{Resultados}

Foi conduzida uma avaliação do VITA com alunos do curso de Bacharelado em Ciência da Computação. Participaram do experimento 7 alunos de duas disciplinas. As avaliações foram enviadas para diferentes dias de aula. Após as avaliações dos alunos, os comentários textuais foram avaliados por 4 professores do curso. Foram escolhidos professores com bastante experiência em docência. Cada professor classificou o nível de satisfação presente nos comentários numa escala de 0 a 10 . O experimento realizado visou avaliar a proximidade da avaliação do VITA com a feita pelos professores. O grau de satisfação fornecido pela ferramenta foi ajustado para uma escala de satisfação VITA $\left(n_{\text {vita }}\right)$ de 0 a 10. Para efeito de comparação, foi considerado o nível de satisfação identificado pelos professores $\left(n_{\text {prof }}\right)$ como a média das classificações feitas pelos professores.

Por fim, foi calculada a diferença entre as duas avaliações ( $n_{\text {vita }}$ e $n_{\text {prof }}$ ) utilizando a correlação de Pearson [Hauke and Kossowski 2011]. A correlação de Pearson mostra o quão correlacionado estão duas variáveis, apresentando um valor no intervalo $[-1,1]$. Uma variável próxima de 1 possui perfeita correlação com outra, enquanto o valor -1 indica uma perfeita correlação inversa. $O$ valor 0 mostra que as variáveis não se relacionam de forma alguma. $\mathrm{O}$ valor de correlação de Pearson obtido para este cenário foi de 0,58 . Assim, podemos concluir que a ferramenta apresenta resultados preliminares positivos.

\section{Conclusão}

O presente trabalho apresentou um método alternativo de avaliação do ensino por parte dos alunos, visando maior aproximação entre alunos e professores, de modo que o docente tenha uma percepção aprimorada e antecipada do indicador de satisfação dos alunos. A satisfação foi medida por intermédio de dois dicionários bastante utilizados na Análise de Sentimentos: LIWC e ANEW.

Assim, o trabalho proposto mostrou-se bastante viável, tendo resultados preliminares bastante promissores. Além disso, vantagens significativas foram destacadas, como 
VII Congresso Brasileiro de Informática na Educação (CBIE 2018)

Anais do XXIX Simpósio Brasileiro de Informática na Educação (SBIE 2018)

uma avaliação mais precisa aula-a-aula, maior agilidade na avaliação de turmas grandes, e maior liberdade do aluno na expressão de sua opinião sobre a aula.

No entanto, a proposta atinge seu limiar quanto ao modelo de Análise de Sentimentos adotado, já que os experimentos foram realizados com dois dicionários. Com isso, extensões da proposta são perfeitamente possíveis tendo em vista o aperfeiçoamento do modelo de Análise de Sentimentos adotado, quanto à integração do sistema proposto à sistemas já existentes, como por exemplo, o Moodle. Um trabalho em andamento é a utilização do VITA em quatro turmas regulares do curso de Ciência da Computação, abrangendo alunos do segundo ao quinto períodos do curso. Este estudo visa avaliar sua influência do VITA na condução das aulas.

\section{Referências}

[Aung and Myo 2017] Aung, K. Z. and Myo, N. N. (2017). Sentiment analysis of students' comment using lexicon based approach. In IEEE/ACIS International Conference on Computer and Information Science (ICIS), pages 149-154.

[Balahadia et al. 2016] Balahadia, F. F., Fernando, M. C. G., and Juanatas, I. C. (2016). Teacher's performance evaluation tool using opinion mining with sentiment analysis. In IEEE Region 10 Symposium (TENSYMP), pages 95-98.

[Barrón-Estrada et al. 2017] Barrón-Estrada, M. L., Zatarain-Cabada, R., Oramas-Bustillos, R., and González-Hernández, F. (2017). Sentiment analysis in an affective intelligent tutoring system. In IEEE International Conference on Advanced Learning Technologies (ICALT), pages 394-397.

[Bradley et al. 1999] Bradley, M. M., Lang, P. J., Bradley, M. M., and Lang, P. J. (1999). Affective norms for english words (anew): Instruction manual and affective ratings.

[Chen et al. 2009] Chen, Y.-L., Hu, H.-W., and Tang, K. (2009). Constructing a decision tree from data with hierarchical class labels. Expert Syst. Appl., 36(3):4838-4847.

[de Souza and Reinert 2010] de Souza, S. A. and Reinert, J. N. (2010). Avaliação de um curso de ensino superior através da satisfação/insatisfação discente. Avaliação: Revista da Avaliação da Educação Superior, 15(1).

[Elliott and Shin 2002] Elliott, K. M. and Shin, D. (2002). Student satisfaction: An alternative approach to assessing this important concept. Journal of Higher Education Policy and Management, 24(2):197-209.

[Filho et al. 2013] Filho, P. P. B., Pardo, T. A. S., and Aluísio, R. M. (2013). An evaluation of the brazilian portuguese liwc dictionary for sentiment analysis. In Brazilian Symposium in Information and Human Language Technology, pages 215-219.

[Gonçalves et al. 2013] Gonçalves, P., Araújo, M., Benevenuto, F., and Cha, M. (2013). Comparing and combining sentiment analysis methods. In Proceedings of the First ACM Conference on Online Social Networks, pages 27-38.

[Hassenzahl 2008] Hassenzahl, M. (2008). User experience (ux): Towards an experiential perspective on product quality. In Proceedings of the 20th Conference on L'Interaction Homme-Machine, pages 11-15.

[Hauke and Kossowski 2011] Hauke, J. and Kossowski, T. (2011). Comparison of values of pearson's and spearman's correlation coefficients on the same sets of data. 30:87-93. 
VII Congresso Brasileiro de Informática na Educação (CBIE 2018)

Anais do XXIX Simpósio Brasileiro de Informática na Educação (SBIE 2018)

[Kao and Poteet 2007] Kao, A. and Poteet, S. R. (2007). Natural language processing and text mining. Springer Science \& Business Media.

[Liu 2012] Liu, B. (2012). Sentiment analysis and opinion mining. Synthesis lectures on human language technologies, 5(1):1-167.

[Manhães et al. 2011] Manhães, L. M. B., da Cruz, S. M. S., Costa, R. J. M., Zavaleta, J., and Zimbrão, G. (2011). Previsão de estudantes com risco de evasão utilizando técnicas de mineração de dados. In Brazilian Symposium on Computers in Education (SBIE), volume 1.

[Ortigosa et al. 2014] Ortigosa, A., Martin, J., and Carro, R. (2014). Sentiment analysis in facebook and its application to e-learning. 31:527-541.

[Pong-Inwong and Kaewmak 2016] Pong-Inwong, C. and Kaewmak, K. (2016). Improved sentiment analysis for teaching evaluation using feature selection and voting ensemble learning integration. In IEEE International Conference on Computer and Communications (ICCC), pages 1222-1225.

[Rani and Kumar 2017] Rani, S. and Kumar, P. (2017). A sentiment analysis system to improve teaching and learning. Computer, 50(5):36-43.

[Robinson et al. 2013] Robinson, R. L., Navea, R., and Ickes, W. (2013). Predicting final course performance from students' written self-introductions: A liwc analysis. Journal of Language and Social Psychology, 32(4):469-479.

[Rodrigues and Guedes 2017] Rodrigues, R. G. a. and Guedes, G. P. (2017). A hybrid affective lexicon for brazilian portuguese. In Proceedings of the XVI Brazilian Symposium on Human Factors in Computing Systems, pages 52:1-52:4.

[Rude et al. 2004] Rude, S., Gortner, E.-M., and Pennebaker, J. (2004). Language use of depressed and depression-vulnerable college students. 18:1121-1133.

[Seldin 1993] Seldin, P. (1993). The use and abuse of student ratings of professors. The Chronicle of Higher Education.

[Tausczik and Pennebaker 2010] Tausczik, Y. R. and Pennebaker, J. W. (2010). The psychological meaning of words: Liwc and computerized text analysis methods. Journal of language and social psychology, 29(1):24-54.

[Vallejos and Steel 2014] Vallejos, C. and Steel, M. (2014). Bayesian survival modelling of university outcomes.

[Weerasinghe et al. 2017] Weerasinghe, I. S., Lalitha, R., and Fernando, S. (2017). Students satisfaction in higher education literature review. American Journal of Educational Research, 5(5):533-539.

[Young 1988] Young, H. P. (1988). Condorcet's theory of voting. American Political Science Review, 82(4):1231-1244.

[Yu et al. 2016] Yu, L.-C., Lee, L.-H., Hao, S., Wang, J., He, Y., Hu, J., Lai, K. R., and Zhang, X. (2016). Building chinese affective resources in valence-arousal dimensions. In Proceedings of the 2016 Conference of the North American Chapter of the Association for Computational Linguistics: Human Language Technologies, pages 540-545. Association for Computational Linguistics. 